\title{
Anticipating the Economic Benefits of Blockchain
}

\author{
Melanie Swan
}

\author{
"In the future, it might seem just as strange to say") \\ that I am trusting a third-party institution with my \\ interests as to say that I'm using an abacus today. \\ Gavin Wood \\ Ethereum Co-Founder
}

\begin{abstract}
In this general overview article intended for non-experts, I define blockchain technology and some of the key concepts, and then I elaborate four specific applications that highlight the potential economic benefits of digital ledgers. These applications are digital asset registries, blockchains as leapfrog technology for global financial inclusion, long-tail personalized economic services, and net settlement payment channels. I also highlight key challenges that offset the potential economic benefits of blockchain distributed ledgers, while arguing that the benefits would outweigh the potential risks. The overarching theme is that an increasing amount of everyday operations involving money, assets, and documents could start to be conducted via blockchain-based distributed network ledgers with cryptographic security, and at more granular levels of detail. One economic implication of widespread blockchain adoption is that the institutional structure of society could shift to one that is computationally-based and thus has a diminished need for human-operated brick-and-mortar institutions.
\end{abstract}

\section{Introduction}

Blockchain (distributed ledger technology) is a network software protocol that enables the secure transfer of money, assets, and information via the Internet, without the need for a third-party intermediary such as a bank (Swan, 2015). Transactions are validated, executed, and recorded chronologically in an appendonly tamper-resistant database, where they remain available on the Internet for on-demand lookup and verification. A digital money system such as Bitcoin is the first and perhaps the most obvious application of blockchain technology. Money can be transferred immediately in real-time from one continent to another, at very low costs, and in a matter of seconds or minutes, instead of waiting days or weeks, and paying high commissions, as is the case with current international money transfer and remittance solutions. Just as the simple mail transfer protocol (SMTP) constitutes the underlying protocol by which Internet users can send an email to each other in a seamless and interoperable way, regardless of their email provider, likewise, the Bitcoin protocol allows people to seamlessly transfer money to one another, regardless of their bank. However, digital currency is but one application enabled by blockchain technology. The four main kinds of applications in development are real-time money transfer and payments, property registries, contractual agreements, and identity confirmation.

The terms blockchain and distributed ledger technology are often used interchangeably. Distributed ledger is the general form of the technology, and blockchain is a specific form with an additional technical detail. Both refer to the concept of a ledger- a file that keeps track of who owns what. A distributed ledger has four salient features: i) a transaction database shared among network members that is ii) updated by consensus, with iii) records timestamped with a unique cryptographic signature, maintained in a iv) tamper-proof auditable history of all transactions. Blockchain adds the additional feature of sequential updating of database records per chained cryptographic hash-linked blocks (each block calls a hash of the previous block, effectively linking 


\section{Anticipating the Economic Benefits of Blockchain}

\section{Melanie Swan}

transaction blocks into an immutable chain, hence the term "blockchain"). There are two kinds of blockchains: public and private. Anyone may use public blockchains such as Bitcoin (bitcoin.org) and Ethereum (ethereum.org), and identity is not known. Private blockchains are analogous to a corporate intranet, used by industry consortia and governments, where users are known and credentialled.

Cybersecurity could be one of the biggest drivers of blockchain adoption. Recent breaches - the private data of an estimated 145.5 million Americans was exposed by the Equifax credit attack (Cowley, 2017) and, in another major attack, the names, emails, and passwords of all 3 billion Yahoo user accounts were stolen (Larson, 2017) - underline the urgent need for better cybersecurity solutions. Centralized databases provide an attractive target for hackers, whereas it is possible that decentralized storage records protected by cryptographic signatures on blockchains might dramatically improve network cybersecurity. Greater user control and permissioning of personal data is an expected feature of decentralized solutions. Blockchains are called "trustless" in that they eliminate having to trust any third party institution such as Equifax or Yahoo in the middle of transactions with personal data. Blockchains are smart networks that confirm and transfer value directly, without third-party intermediaries. Intelligence is built directly into the network's operations through a sophisticated protocol that automatically identifies, validates, confirms, and routes transactions within the network. The result is a trustless system in that the human counterparties and institutions involved do not need to be known and trusted. Instead, trust is placed in the computational smart network system, which could help to create next-generation cybersecurity solutions.

\section{Application 1. Digital Asset Registries}

Following digital currencies and money transfer, one of the biggest blockchain applications in development is digital asset registries. The same distributed ledger technology provides the means to record and transmit digital goods over the Internet, while ensuring that these goods cannot be copied or multiplied (thereby addressing the double-spending problem that has been an issue with digital currencies previously). A digital asset registry is a listing of smart assets (also referred to as smart property). A smart asset is an asset that is registered to a blockchain and thus can be easily verified and transferred because of this digital registration (Swan \& de Filippi, 2017). Digital asset registries might use blockchains extensively as a system to record, transfer, and verify asset ownership. This could include titles for automobiles, homes, and land.

Land titling systems are a "low hanging fruit" application to demonstrate blockchains in practical use. Some countries have pilot programs underway, notably Georgia, Ukraine, Sweden, and Ghana (Reese, 2017). In Sweden, the government estimates that the project could save taxpayers over $\$ 106$ million USD a year by eliminating paperwork, reducing fraud, and speeding up transactions (Lantmäteriet, 2016; Wong, 2017). The money at stake suggests resistance to new solutions, for example, the United States title insurance industry earns $\$ 18$ billion USD a year for a product that some have evaluated as outdated and largely unneeded, even before the concept of blockchain-based registries (Woolley, 2006). There are property transfer issues and also legal implications. A blockchain can be used as a digital registry to record, transfer, and verify asset ownership (home, auto, stocks, bonds, mortgages, and insurance), and also to preserve the integrity and authenticity of sensitive documents or records (e.g., passports, visas, driver's licenses, birth and death certificates, voter registration, contracts, wills, patents, and medical records). An exemplar implementation of digital asset registries for identity services is the State of Illinois's blockchainbased birth registry project (Illinois Blockchain Initiative, 2017).

\section{Application 2. Leapfrog Technology}

One of the highest-impact applications of blockchains could be as a leapfrog technology for global financial inclusion. It does not make sense to build out brick-andmortar bank branches to every last mile in a world of digital services. Instead, eWallet banking apps might be an effective means of reaching the two billion "unbanked" people in the world (PwC, 2016). Even without phonebased banking, low-cost debit cards might effectively service the unbanked (Rogoff, 2016). These kinds of "FinTech "solutions (i.e., financial technology: financial services delivered by technology) could have the benefit of opening up new markets to service providers who did not have a cost-effective method of addressing these customers previously (Swan, 2017). The leapfrog impact could be significant as banking services are bundled together with identity services and land registries. The United Nations estimates that 1.1 billion people, one sixth of the world's population, live without an officially recognized identity (2017). Similarly, the World Bank estimates that $70 \%$ of the world's population lacks access 


\section{Anticipating the Economic Benefits of Blockchain}

\section{Melanie Swan}

to land titling (Heider \& Connelly, 2016). Land titling and property transfer systems have been identified as a crucial step for economic development (de Soto, 2003). The adoption challenges are perhaps not always technical as much political given that solutions are only possible to the extent that power elites are willing to implement them (Chua, 2004).

A demonstration case of digital financial services as a leapfrog technology is the mobile payments market in China. In 2016, Chinese people and organizations spent the equivalent of $\$ 5.5$ trillion USD through mobile payment platforms, about 50 times the amount spent in the United States (Kuhn, 2017). Nearly half $(42.4 \%)$ of in-store purchases in China are via non-cash payments (Chen, 2017). Debit cards and credit cards were not offered and adopted in China to the same extent as in other countries, and thus an alternative to cash such as mobile payment has been widely adopted. More broadly, credit is in some sense a "luxury service" only extended to a small percentage of people worldwide. In an era of blockchain-based digital finance, credit could be a consumer service that is much more transparent, widely available, and synchronized across country boundaries. For example, there could be open-source FICO scores, blockchain-based credit bureaus, and blockchains as the backbone of the first international credit agency (Swan, 2016). Just as blockchain-based electronic medical records can be accessed securely anywhere in the world, so too could credit scores. The impact could be opening up credit markets to retail customers on a global basis. There could be advantages such as individuals not having to build credit histories from scratch when living in a different country. But there could also be drawbacks, as not everyone might want to join a global credit system (although one that is more transparent and user-controlled might be more welcome).

\section{Application 3. Long-Tail Personalized Economic Services}

The long-tail argument is that, in digital marketplaces, it is possible to sell lower quantities of more items (Anderson, 2008). The $80 / 20$ rule - the classic logic that $80 \%$ of sales come from the top-selling $20 \%$ of items does not hold in digital marketplaces. Researchers confirm long-tail economics in digital marketplaces, finding that niche books account for $36.7 \%$ of Amazon's sales (Brynjolfsson et al., 2010). They argue that power laws as opposed to Pareto distributions are a better model for digital marketplace sales for books, music, and software downloads. For the blockchain economy, the key point is that not only are long-tail markets economically viable, but also that there is demand for personalized products and services that cater to individual needs. Previously, one size had to fit all in financial and government services due to economies of scale and other barriers. However, in a network economy with blockchain-based asset transfer, personalized financial and government services might be better tailored to individual needs and wants. An example of personalized economic services where one size does not fit all is that, instead of a standard 30-year mortgage, a borrower might prefer a 22-year mortgage that better corresponds to personal life events such as a planned home downsizing once children are grown.

Amazon, eBay, and Craigslist are digital marketplaces that allow the long tail of economics to meet in the sense of the buyer of a particular rare item being able to find a seller of that item in a way that would not be possible in a mass-market retail store. The point is that, in digital marketplaces, buyers and sellers can transact more granular personalized business than is economically feasible in the brick-and-mortar format. Likewise, with blockchains, the long tail of personalized financial services might be able to meet in "eBay for money" type websites, where the buyer of a specific financial service could find a provider. The implication of algorithmic trust, and funds locked or escrowed with smart contracts, is that any two long-tail parties can meet and transact in a secure blockchain-based environment, without having to know each other. Personalized banking, credit, and financial services could become routine, and also personalized governance services, for example, a closer link between the public services funded and consumed by individuals. Early evidence of long-tail markets for blockchain services is a September 2017 transaction that purports to be the first real asset transfer with a blockchain. US-based TechCrunch founder Mike Arrington purchased a Ukraine-based residence using Propy, a global decentralized property store on the Ethereum blockchain (Masse, 2017). This notable transaction strikes a parallel with Meg Whitman's automobile purchase on the eBay Motors website in the early 2000s, which helped to legitimize digital marketplaces for large-size transactions.

\section{Application 4. Payment Channels and Peer Banking Services}

One of the most intriguing ideas being developed in the blockchain industry is payment channels. A payment channel is a financial contract executed over time in three steps: i) one party opens up a payment channel 


\section{Anticipating the Economic Benefits of Blockchain}

\section{Melanie Swan}

with one or more parties and posts a pre-payment escrow balance on file, ii) the party consumes against this credit over time, until iii) the closing transaction in which aggregate activity is booked in one net transaction to close the contract. The idea arose for micropayments, such as video bandwidth consumption, where piecemeal transactions do not make sense and an automated contractual arrangement can support aggregate consumption. Payment channels are similarly conceived for regular consumption such as opening up a Starbucks payment channel for $\$ 50$ each month. The daily coffee consumed is tracked and booked against the $\$ 50$ channel and netted at the end of the month. Contracts close and roll over at regular intervals. Either party may elect to close the payment channel early, in which case the net settlement would be booked and the contract would end. Another benefit of payment channels is easing blockchain scalability by only booking the opening contract and the final amount as opposed to interim transactions, while being contractually obligated and protected all along the way.

Payment channels are a speculative concept that is under discussion, but the conceptual implications are provocative. First, the radical implication of peer-to-peer networks is that any node can deliver services to other nodes, for a small transaction fee. This is already how the Bitcoin network operates, with 9,352 worldwide peer nodes (bitnodes.21.co) hosting the transaction ledger. The mining operation to confirm and log transactions is another network peer-based activity. Storage and news hosting are newer network services, and the implication is that payment channels have the requisite functionality to allow peer nodes to offer banking services (Dryja, 2016). We start to see what the claim that cryptocurrencies are "programmable money" might actually mean in implementation. Recognizable feature sets from other financial contracts (for example prepayment risk and European/American-style option execution) can be enabled easily in blockchain-based contracts such as payment channels. The question arises as to how to treat payment channels from an accounting and legal standpoint. For accounting purposes, is a payment channel a deferred payment or an installment sale? When during the contract is revenue to be recognized, and what are the balance sheet liability obligations? Legally, do payment channels constitute assignments of claims or forward-looking IOUs? A contingent three-part financial contract over time is a new instrument, especially when considering that transfers might exist across multiple hops (parties) in a directed graph structure of layered contingencies that is based on distributed computing network architectures as opposed to traditional modes of financial exchange.

Other conceptual implications are similarly striking. The idea of economic activity based on net settlement versus gross settlement is intriguing. Despite the current limit of roughly $\$ 175$ (4\% of the value of one Bitcoin) placed on the total payment channel transfer amount of any one channel by the existing solution provider, the Lightning Network (lightning.network), other payment channel solutions could have different parameters. What if many more operations in the economy were to transition to a net payments basis? Central banks clear amongst themselves with real-time gross settlement (RTGS) systems, as does Ripple (ripple.com). Industry consortia such as interbank daily settlements are tabulated on a net basis. However, what about opening up net clearing functionality to individuals? The idea is essentially an enhanced version of paycheque direct deposit plus auto-pay bills, just formalized into a multi-party payment contract. Personal monthly inflows and outflows could be orchestrated by a payment contract that nets salary against expenses and builds in a savings remainder. With money and payments digitized, and activity being securely forwardcommitted by payment contracts, the implication is that net flows instead of gross flows might be transferred. An economy based on net clearings or contracts for difference is quite different than the current system, and the risks and benefits would need to be evaluated.

A further implication of digitized money and payments is that the standard amounts at which we do business could be much more granular. This granularity could possibly allow progress in reconceiving the debt juggernaut impacting individuals and institutions alike. Streaming money could be disgorged in much smaller chunks that are more closely tied to costs and repayment possibilities (Antonopoulos, 2017). We could similarly reconceive economic modes of consumption and the related financing options. There could be a reconstitution of mechanisms for pre-paid consumption (a small part of current overall economic activity) against the much larger portion of activity that is post-paid and based on credit and terms. Digitized streaming money and payment channels could be techniques to quicken the 30-60-90 day terms and uncollectible debt problem in supply chain finance, and facilitate a just-in-time economy for money. 


\title{
Anticipating the Economic Benefits of Blockchain
}

\author{
Melanie Swan
}

\section{Key Challenges}

The potential economic benefits of blockchain distributed ledgers are offset with several key challenges. The first challenge is that the technology is complicated. Even the basics are difficult to understand, both conceptually and technically, and this is a barrier to effective decision making and the ongoing implementation and use of the technology. Second, a variety of challenges have to do with the technical aspects of the technology. Bitcoin is the largest open blockchain in a field where private blockchains are also starting to be in use. As the biggest demonstration case, one concern specific to Bitcoin is that $70 \%$ of the mining operation (data centres that validate and confirm transactions) is concentrated in China (Coleman, 2016). Others counter that this is not a long-term risk as the mining operation would likely become more globally diversified as the use of the currency grows. Another concern is that there seem to be a variety of unresolved technical issues. However, it is important to note that Bitcoin is an open source software project, and that it is helpful, not detrimental, that all of the issues are publicly debated by worldwide developers. So far, changes to the software have been proceeding democratically, with all network participants involved in decision making (the five big constituencies are developers, miners, exchanges, wallets, and merchants). A recent example of this is the hard fork for the Bitcoin blockchain to incorporate SegWit2x, a new standard increasing scalability, with the decision made in August 2017 for implementation in November 2017 (Higgins, 2017). Others worry about hacking scandals, and these would likely persist in the ongoing development of blockchain ledger systems, while cybersecurity responses will also continue to develop in lockstep.

The third challenge is scalability - creating distributed financial networks that can scale to Visa-class processing levels and beyond - given that the networks may be used for a wider variety of transaction types. By comparison, Visa processes an average volume of 1,667 transactions per second, and Bitcoin processes 7 per second (Vermeulen, 2017). Visa transfers $\$ 18$ billion per day, and Bitcoin transfers $\$ 300$ million per day. At the heart of the issue is coordinating the operation of distributed computing networks. Visa is a closed proprietary network. Other computing networks that are more similar to distributed ledgers are those such as Google's PageRank. This system is distributed, but has an overall control mechanism (a locking service called Chubby in Google's case) that coordinates how network nodes are updated.
Public blockchains are different in that they are truly open distributed networks which any new peer may join. The computer science problem is getting 1 million distributed clients to agree, including when there could be malicious nodes on the network, because it is open (Williams, 2016). The mechanism by which distributed systems come to agreement about new truth states of the network is called consensus (and relatedly Byzantine agreement). The concept is that of a "world computer" that is securely and efficiently coordinated by algorithms as to how the network reaches consensus on new truth states of the database (ledger) and nodes update their copy. The kinds of consensus algorithms currently in use by blockchain systems are "proof of work" and "proof of stake". However, these algorithms may not be scalable solutions for the longer term. Proof of work, while secure, is inefficient. One estimate is that the Bitcoin network could consume as much electricity as Denmark by 2020 (Deetman, 2016) due to the proofof-work requirement. Even though private blockchains have known and credentialled users, their scalability, too, is gated by the ability to update nodes in very large distributed networks. Thus, the development of consensus algorithms that are scalable, efficient, and secure is a challenge for the long-term viability of blockchain technology.

The fourth challenge is effective government regulation to support the development of the industry. Because blockchains deal with money (a sensitive area with incentive for corruption), they are likely to continue to be under the purview of national regulation. Although the technology allows anyone to download software and set themselves up as a bank, offering financial services on a payment gateway, it is not legal to do so. Even the most basic exchange between cryptocurrencies and fiat currency is deemed a money transmitter service and must be appropriately licensed by state agencies in order to be legal in the United States (Lujan, 2017). The onus is on entrepreneurs to determine the relevant regulatory aspects that apply to their businesses and then either comply or face non-compliance charges.

As with any new technology, the challenge is encouraging honest activity while thwarting nefarious behaviour. With blockchain, the question is how to encourage businesses to explore the new frontier enabled by digital ledgers, while managing an environment that simultaneously invites new kinds of scams and wrongdoing. The most recent example is the "dotcom boom" in initial coin offerings (ICOs), which have raised $\$ 2.7$ billion USD to date, and how different national governments are regulating them (CoinDesk, 


\section{Anticipating the Economic Benefits of Blockchain}

\section{Melanie Swan}

2017). In the United States, ICOs are evaluated on a case-by-case basis, and if they behave like a security, they are deemed as such, and fall under securities regulations (US SEC, 2017). Regarding law enforcement, digital ledgers should be recognized as a new and more complicated digital venue where illegal activities may be taking place alongside honest activities. Regulatory agencies are called upon to become savvy about the risks presented by the new technology and operate within this domain. Regulators need to understand how digital ledger technologies work and can be used for operations such as money laundering, and they need to understand how illegal practices might be detected, tracked, and persecuted in these new transnational cryptographic areas. An example of this was regulators using the tracking features inherent to blockchain transactions to apprehend perpetrators in the Silk Road case (Brandom, 2015). While being cognizant of these and other challenges, overall, the economic benefits of blockchain could outweigh the potential risks.

\section{Conclusion}

Blockchain distributed ledgers have the ability to securely digitize many current operations in economics and finance, and legal and government services, such that they might be reengineered for the Internet era. The four main kinds of blockchain applications are money transfer and payments, property registries, contractual agreements, and identity confirmation. Blockchains are able to transfer money and assets, and also preserve the authenticity of sensitive documents and records. The terms blockchains and digital ledgers are generally interchangeable, although blockchains have an additional feature in that transaction blocks are linked together with cryptographic hashes, which provides additional security. Blockchains could be important in cybersecurity solutions because they have decentralized storage records protected by cryptographic signatures as opposed to centralized databases that attract hackers.

In this article, four specific blockchain applications were examined that might have a positive economic benefit. First considering digital asset registries, there are a number of projects underway, particularly for improved efficiency in land titling and birth registration.
Second, blockchains are identified as an important leapfrog technology for global financial inclusion with eWallet banking services, identity registration, and land titling. Third, distributed ledgers might allow personalized economic services to be created such as non-standard mortgages to suit individual needs in digital marketplaces that are like an "eBay for money." Fourth, a speculative technology called payment channels might eventually develop into a digitized payment system for resource consumption that settles based on net payments instead of gross transfers, and enables peerto-peer banking services. The overarching theme that emerges from this analysis is that many daily operations involving money, assets, and documents could start to be conducted on digital networks with cryptographic security. Given that less friction and human involvement may be needed to transfer goods and services, less physical infrastructure might be needed to make it happen. It is not that the influence and role of institutions would wane, but that their material footprint and how they do business could change substantially in a blockchain economy.

\section{About the Author}

Melanie Swan is a technology theorist in the Philosophy Department at Purdue University in West Lafayette, Indiana, United States. She is the author of the best-selling book Blockchain: Blueprint for a New Economy (2015), which has been translated into six languages. She is the founder of several startups including the Institute for Blockchain Studies, DIYgenomics, GroupPurchase, and the MS Futures Group. Ms. Swan's educational background includes an MBA in Finance and Accounting from the Wharton School of the University of Pennsylvania, an MA in Contemporary Continental Philosophy from Kingston University London and Université Paris 8, and a BA in French and Economics from Georgetown University. She is a faculty member at Singularity University and the University of the Commons, an Affiliate Scholar at the Institute for Ethics and Emerging Technologies, and an invited contributor to the Edge's Annual Essay Question. 


\section{Anticipating the Economic Benefits of Blockchain}

\section{Melanie Swan}

\section{References}

Anderson, C. 2008. The Long Tail: Why the Future of Business is Selling Less of More. New York: Hachette Books.

Antonopoulos, A. 2017. Advanced Bitcoin Scripting. SF Bitcoin Developers Seminar, April 20, 2017. Accessed October 18, 2017: https://www.youtube.com/watch?v=MiS8-4uIOYo

Brandom, R. 2015. In the Silk Road Trial, Bitcoin is a Cop's Best Friend. The Verge, January 14, 2015. Accessed October 18, 2017: https://www.theverge.com/2015/1/14/7546669/silk-road-trialbitcoin-tracking

Brynjolfsson, E., Hu, Y. J., \& Smith, M. D. 2010. The Longer Tail: The Changing Shape of Amazon's Sales Distribution Curve. SSRN, September 20, 2010. Accessed October 18, 2017: http://dx.doi.org/10.2139/ssrn.1679991

Chen, T. 2017. China Mobile Payment Report 2017. WalktheChat, June 25, 2017. Accessed October 18, 2017: https://walkthechat.com/china-mobile-payment-report-2017/

Chua, A. 2004. World on Fire: How Exporting Free Market Democracy Breeds Ethnic Hatred and Global Instability. New York: Anchor Books.

CoinDesk. 2017. CoinDesk ICO Tracker: All-Time Cumulative ICO Funding. CoinDesk, October 13, 2017. Accessed October 18, 2017: https://www.coindesk.com/ico-tracker

Coleman, L. 2016. China's Mining Dominance: Good or Bad For Bitcoin? Cryptocoins News, September 14, 2016. Accessed October 18, 2017:

https://www.cryptocoinsnews.com/chinas-mining-dominancegood-or-bad-for-bitcoin/

Cowley, S. 2017. 2.5 Million More People Potentially Exposed in Equifax Breach. New York Times, October 2, 2017. Accessed October 18, 2017:

https://www.nytimes.com/2017/10/02/business/equifaxbreach.html

Deetman, S. 2016. Bitcoin Could Consume as Much Electricity as Denmark by 2020. Motherboard, March 29, 2016. Accessed October 18, 2017:

https://motherboard.vice.com/en_us/article/aek3za/bitcoincould-consume-as-much-electricity-as-denmark-by-2020

De Soto, H. 2003. The Mystery of Capital: Why Capitalism Triumphs in the West and Fails Everywhere Else. New York: Basic Books.

Dryja, T. 2016. Lightning Network as a Directed Graph: Single-Funded Channel Topology. SF Bitcoin Developers Seminar, April 24, 2016. Accessed October 18, 2017:

https://www.youtube.com/watch?v=-lgYYz3y_hY

Heider, C., \& Connelly, A. 2016. Why Land Administration Matters for Development. World Bank, June 28, 2016. Accessed October 18, 2017:

http://ieg.worldbankgroup.org/blog/why-land-administrationmatters-development

Higgins, S. 2017. Bitcoin Cash Just Mined Its First Block, Making Blockchain Split Official. CoinDesk, August 1, 2017. Accessed October 18, 2017:

https://www.coindesk.com/bitcoin-cash-just-mined-first-blockmaking-blockchain-split-official/
Illinois Blockchain Initiative. 2017. Illinois Partners with Evernym to Launch Birth Registration Pilot. The Illinois Blockchain Initiative, Press Release, August 31, 2017. Accessed October 18, 2017: https://illinoisblockchain.tech/illinois-partners-with-evernym-tolaunch-birth-registration-pilot-f2668664f67c

Kuhn, A. 2017. In China, A Cashless Trend Is Taking Hold With Mobile Payments. NPR, June 29, 2017. Accessed October 18, 2017: http://www.npr.org/sections/alltechconsidered/2017/06/29/5348 46403/in-china-a-cashless-trend-is-taking-hold-with-mobilepayments

Lantmäteriet. 2016. The Land Registry in the Blockchain. Gävle, Sweden: Lantmäteriet. http://ica-it.org/pdf/Blockchain_Landregistry_Report.pdf

Larson, S. 2017. Every Single Yahoo Account Was Hacked - 3 Billion in All. CNN, October 4, 2017. Accessed October 18, 2017: http://money.cnn.com/2017/10/03/technology/business/yahoobreach-3-billion-accounts/index.html

Lujan, S. 2017. U.S. Government Cracks Down on Illegal Bitcoin Money Transmitters. Bitcoin.com, May 5, 2017. Accessed October 18, 2017: https://news.bitcoin.com/government-cracks-down-on-illegalbitcoin-money-transmitters/

Masse, B. 2017. Apartment Purchased with Ethereum Raises Hopes, Questions. Inman, September 29, 2017. Accessed October 18, 2017: https://www.inman.com/2017/09/29/apartment-purchased-withethereum-raises-hopes-questions/

PwC. 2016. The Un(der)banked is FinTech's Largest Opportunity. DeNovo Q2 2016 FinTech ReCap and Funding ReView. New York: PwC Strategy\&.

https://www.strategyand.pwc.com/media/file/DeNovo-QuarterlyQ2-2016.pdf

Reese, F. 2017. Land Registry: A Big Blockchain Use Case Explored. CoinDesk, April 19, 2017. Accessed October 18, 2017 :

https://www.coindesk.com/blockchain-land-registry-solutionseeking-problem/

Rogoff, K. S. 2016. The Curse of Cash. Princeton, NJ: Princeton University Press.

Swan, M. 2015. Blockchain: Blueprint for a New Economy. Sebastopol, CA: O’Reilly Media.

Swan, M. 2016. Decentralized Finance: Blockchains, Prediction, and Valuation. Paper presented at The Economist: Finance Disrupted, New York, NY, October 13, 2016. http://www.financedisrupted.com/melanie-swan/

Swan, M. 2017. Expectation on Blockchain: Blockchain Economics and Finance. Chijo (Intelplace), 121: 17-24. http://melanieswan.com/documents/msj.pdf

Swan, M., \& de Filippi, P. 2017. Toward a Philosophy of Blockchain: A Symposium: Introduction. Metaphilosophy, 48: 603-609. http://dx.doi.org/10.1111/meta.12270

United Nations. 2017. ID2020 Summit. United Nations. Accessed October 18, 2017: http://id2020summit.org

US SEC. 2017. SEC Issues Investigative Report Concluding DAO Tokens, a Digital Asset, Were Securities. United States Securities and Exchange Commission (US SEC), Press Release, July 25, 2017. Accessed October 18, 2017: https://www.sec.gov/news/press-release/2017-131 


\section{Anticipating the Economic Benefits of Blockchain}

\section{Melanie Swan}

Vermeulen, J. 2017. Bitcoin and Ethereum vs Visa and PayPal Transactions Per Second. My Broadband, April 22, 2017. Accessed October 18, 2017: https://mybroadband.co.za/news/banking/206742-bitcoin-andethereum-vs-visa-and-paypal-transactions-per-second.html

Williams, D. 2016. DFINITY: Applications of Verifiable Random Function. Stanford Computer Forum, November 15, 2016. Accessed October 18, 2017:

https://www.youtube.com/watch?v=hX0DkgQloDE

Woolley, S. 2006. Inside America's Richest Insurance Racket. Forbes, October 28, 2016. Accessed October 18, 2017:

https://www.forbes.com/forbes/2006/1113/148.html

Wong, J. I. 2017. Sweden's Blockchain-Powered Land Registry Is Inching towards Reality. Quartz, April 3, 2017. Accessed October 18, 2017:

https://qz.com/947064/sweden-is-turning-a-blockchain-poweredland-registry-into-a-reality/

Citation: Swan, M. 2017. Anticipating the Economic

Benefits of Blockchain. Technology Innovation

Management Review, 7(10): 6-13.

http://doi.org/10.22215/timreview/1109

Keywords: blockchain, economics, payment channels, distributed ledgers, cryptocurrencies, cybersecurity,

cryptoeconomics, long tail markets, digital goods, smart assets, smart contracts, streaming money,

programmable money, lightning network, digital asset registries, eWallet 


\section{Academic Affiliations and Funding Acknowledgements}
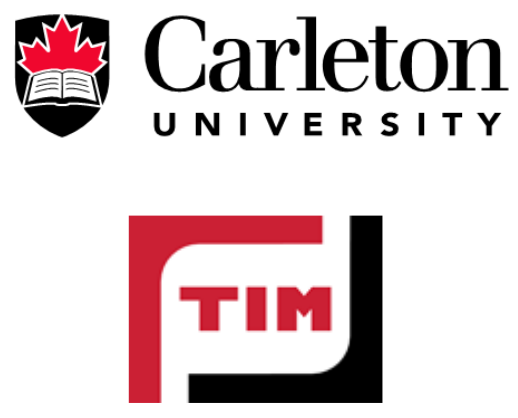

Canadà
Technology Innovation Management (TIM; timprogram.ca) is an international master's level program at Carleton University in Ottawa, Canada. It leads to a Master of Applied Science (M.A.Sc.) degree, a Master of Engineering (M.Eng.) degree, or a Master of Entrepreneurship (M.Ent.) degree. The objective of this program is to train aspiring entrepreneurs on creating wealth at the early stages of company or opportunity lifecycles.

- The TIM Review is published in association with and receives partial funding from the TIM program.

The Federal Economic Development Agency for Southern Ontario (FedDev Ontario; feddevontario.gc.ca) is part of the Innovation, Science and Economic Development portfolio and one of six regional development agencies, each of which helps to address key economic challenges by providing regionallytailored programs, services, knowledge and expertise.

- The TIM Review receives partial funding from FedDev Ontario's Investing in Regional Diversification initiative. 